\title{
Application of SVM Based on Improved Grid Search in Quantitative Analysis of $\mathrm{CO}_{2}$ Gas
}

\author{
Jian $\mathrm{Qu}^{1, \mathrm{a}}$, Hongyan Chen ${ }^{2, \mathrm{~b}^{*}}$, Wenzhen Liu ${ }^{3, \mathrm{c}}$, Bing Zhang ${ }^{4, \mathrm{~d}}$, Zhibin $\mathrm{Li}^{5, \mathrm{e}}$ \\ $1,2,3,4,5$ College of Mechanical and Electrical Engineering, China Jiliang University, China \\ a 13645712326@163.com, ${ }^{\mathrm{b}^{*}}$ bbchy@163.com, ${ }^{\mathrm{c}}$ 848990467@qq.com, ${ }^{\mathrm{d}}$ zhangbingapple@qq.com \\ e1247139378@qq.com
}

Keywords: SVM; grid search; gas sensor; exhaust emission.

\begin{abstract}
According to the difficult in selecting parameter of SVM when modeling on the gas quantitative analysis, and existing methods need long time,SVM optimized by improved grid search method was proposed to built an infrared spectrum quantitative analysis of gas.According to this method, the spectrum data of $\mathrm{CO}_{2}$ is optimized.The kernel function leads SVM and calculate the concentration.By using improved grid search, quantitatively analyzed 18 different concentrations of $\mathrm{CO}_{2}$ in the range between $1 \% \sim 25 \%$. The experiment results show that this method gets $\mathrm{c}=1, \mathrm{~g}=1, \mathrm{cvMSE}=0.00413$. And the prediction error is less than $4 \%$, in line with national standards for exhaust emissions.
\end{abstract}

\section{Introduction}

Auto exhausted emission has become an important factor of environmental pollution, the pollution gases of exhausted emission are mainly $\mathrm{CO}, \mathrm{NO}, \mathrm{CO}_{2}, \mathrm{HC}$ compounds and so on. $\mathrm{CO}_{2}$ is a greenhouse gas, excess emissions will seriously affect our living environment. To control the automobile vehicle emissions, it must be started with the sources of pollution and enhance the detection and control of motor vehicle exhaust emissions. The demand of stable and accurate gas analytical systems has become very urgent.In the quantitative analysis of $\mathrm{CO}_{2}$, infrared absorption is the main analysis method .But due to the factors of aging sensor components, the change of temperature and power supply voltage fluctuation,leads to the measurement accurancy is not high.

This paper is based on SVM[1][2] in the concentration range of $1 \% \sim 25 \%$ of 18 samples in $\mathrm{CO}_{2}$ gas for quantitative analysis, and in the selected issues of SVM parameters, in order to reduce the average relative error further in quantitative analysis, reduce the optimization time and get the best parameter combination, the improved grid parameters optimization method is put forword and the optimal prediction model is set up.

\section{Theory of SVM}

In this paper, we take a known concentration of $\mathrm{CO}_{2}$ gas samples as the training set of, $T=\left\{\left(x_{1}, y_{1}\right), \ldots,\left(x_{N}, y_{N}\right)\right\} \in\left(R^{n} \times R\right)^{N}$ and $x_{i} \in R^{n}$ is i-th sample of tested $\mathrm{CO}_{2}$ gas, $x_{i}=\left(x_{1}, x_{2}, \cdots, x_{L}\right)$ is $\mathrm{L}$ spectral datas within the scanning range of the wavelength, $y_{i} \in R$ is the concentration of tested $\mathrm{CO}_{2}$ gas, $\mathrm{i}=1,2, . ., \mathrm{N}$. In the highdimensional space, the regression function $f\left(x_{i}\right)$ of spectrum needed to solve and the concentration of $\mathrm{CO}_{2}$ gas can be expressed as follows:

$$
f\left(x_{i}\right)=\omega \cdot \phi\left(x_{i}\right)+b
$$

Among them, $\omega \cdot \phi\left(x_{i}\right)$ is the inner product of $\omega$ and $\phi\left(x_{i}\right) ; \omega$ is regression coefficients; $b$ is threshold.

According to SRM, the formula (1) is converted to a convex quadratic programmin problem:

$$
\min _{\omega, \xi} \frac{1}{2} \omega^{T} \omega+C \sum_{i=1}^{N}\left(\xi+\xi^{*}\right)
$$


And the regression function of SVM regression model is:

$$
f(x)=\omega \cdot \phi(x)+b=\sum_{i=1}^{N}\left(\alpha_{i}-\alpha_{i}^{*}\right) k\left(x_{i}, x_{j}\right)+b
$$

In formula (3), if $\alpha_{i}$ is not zero or $\alpha_{i}{ }^{*}$ is not zero, indicates that this sample is the support vector.

Commonly used kernel functions have linear kernel, polynomial kernel function, radial basis (RBF) kernel function, Sigmoid Kernel. Because of Gauss function can nicely simulate a spectrum signal, so we select of RBF kernel function[3] in this paper.

\section{The improved grid search method}

The estiblishment of SVM regression model need to optimize the parameters, and the main parameters are $\mathrm{c}$ and g.First of all, a long step cursory search is used in a large range, thus a group of $\mathrm{c}$ and $\mathrm{g}$ of the highest accuracy of classification is selected.After the local optimal parameters are found and an inter cell in the vicinity of the set of parameters selected.As the second fine search,the small step of traditional method is used to find the final optimal parameters. The concrete optimizing procession can be expressed as shown in Figure 1.

\section{Experiment and Analysis}

In this paper, $\mathrm{CO}_{2}$ concentration is detected by the application of nondispersive infrared method (NDIR).It could rapidly measure the concentration of carbon dioxide, carbon monoxide and hydrocarbon in the emission exhaust at the same time. The infrared light which is modulated by MCU go through a certain length of the gas chamber, which is filled with $\mathrm{CO}_{2}$ gas concentration measured.

After a specific wavelength light source through the gas, due to the $\mathrm{CO}_{2}$ 's absorption of infrared energy in the appointed wavelength of infrared band, after the light passes through, in the corresponding line of the light intensity attenuation will occur, the infrared energy will be reduced, the probe detects the remaining light intensity and converts into electrical signals, amplified and filtered as model input .

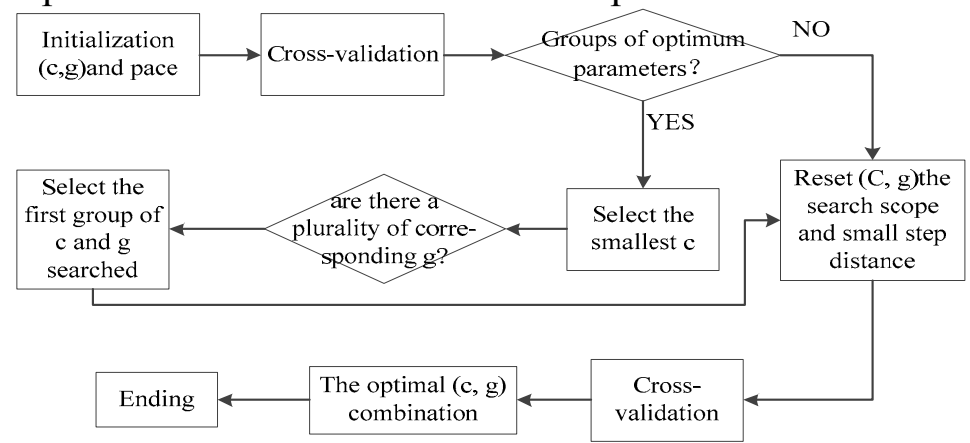

Fig.1 The flow chart of improved grid search optimization Principle of the test system is shown in Figure 2:

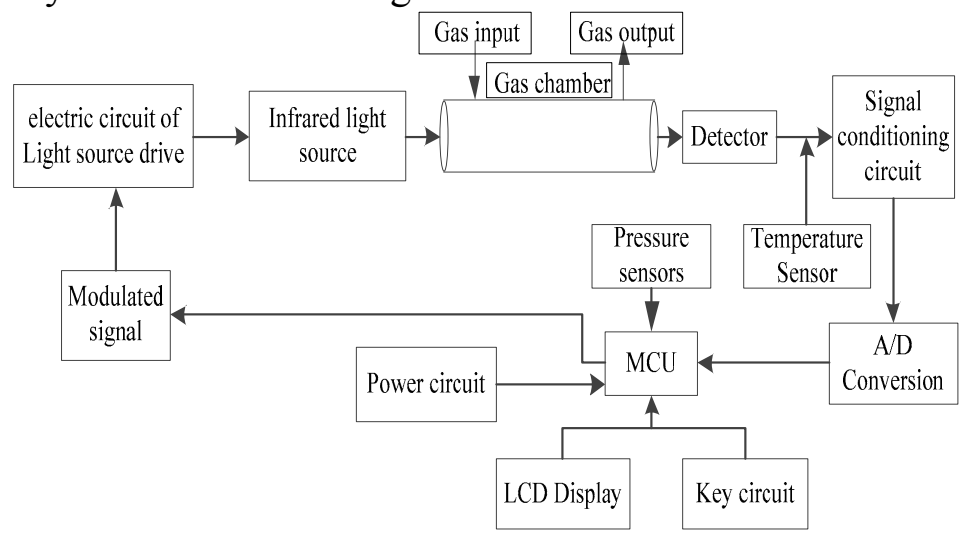

Fig.2 The NDIR test system schematic 
Standard concentration between $1 \% \sim 25 \%$ of the 18 groups of $\mathrm{CO}_{2}$ gas as the experimental sample gas, the concentration of the standard were normalized, that is the desired output of the model. Model needs to find the optimal penalty parameter $\mathrm{c}$ and RBF kernel parameter $\mathrm{g}$, In this paper, we apply a method of grid search to optimize parameter. The basic principle of grid search method is to make $\mathrm{c}$ and $\mathrm{g}$ meshing and through all points in the grid in a certain range and get values, for given $\mathrm{c}$ and $\mathrm{g}$, calculate the accuracy of classification of training set taking advantage of $\mathrm{K}-\mathrm{CV}$ [4] under this group $\mathrm{c}$ and $\mathrm{g}$, and ultimately taking $\mathrm{c}$ and $\mathrm{g}$ that make the highest classification accuracy of the training set as the best parameters.

Model needs to find the optimal penalty parameter $\mathrm{c}$ and RBF kernel parameter $\mathrm{g}$. In this paper, we apply a method of grid search[5] to optimize parameter. The results are shown in Figure 3.

Well selected optimal parameter combination (c, g), the SVM regression model can be established for standard sample data, so that you can treat the measured gas sample regression analysis.

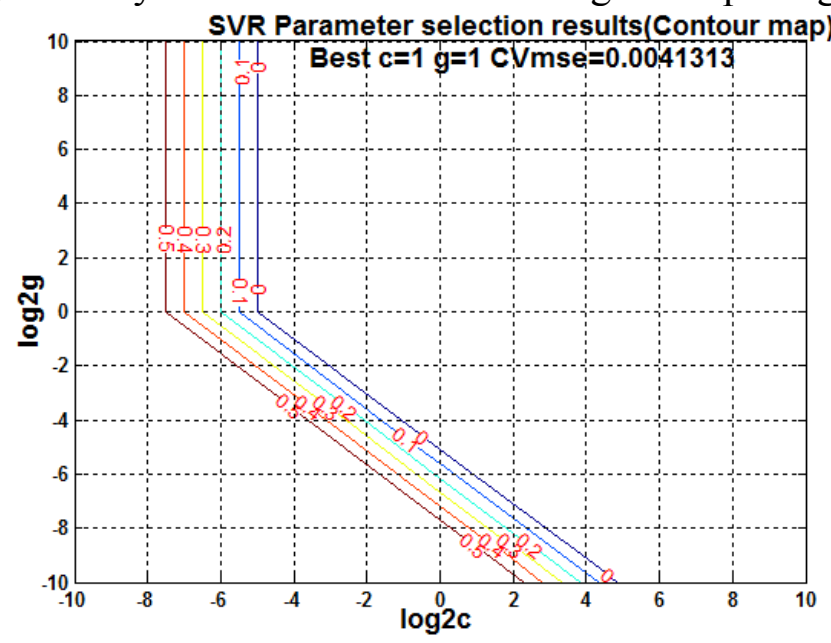

Fig.3 Parameter selection results

The comparison chart of actual output and expected output of the model, as well as the relative error are shown in Fig 4 and Fig 5.

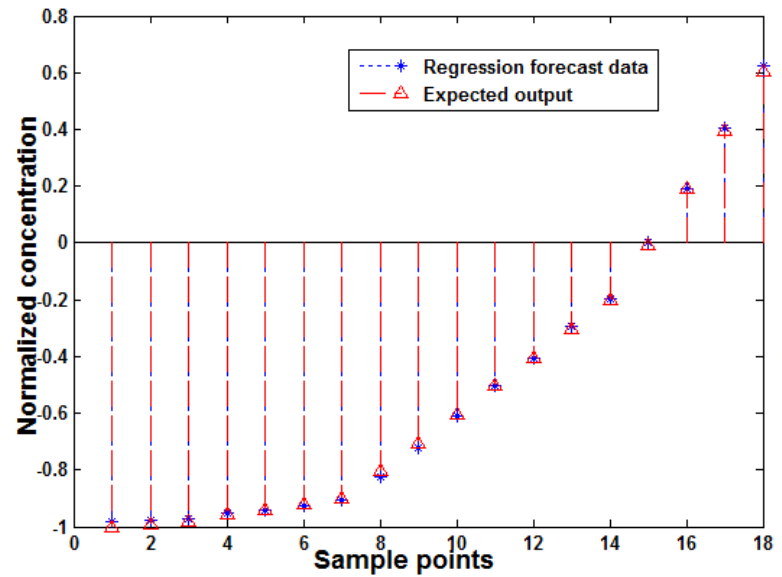

Fig.4 The comparison chart

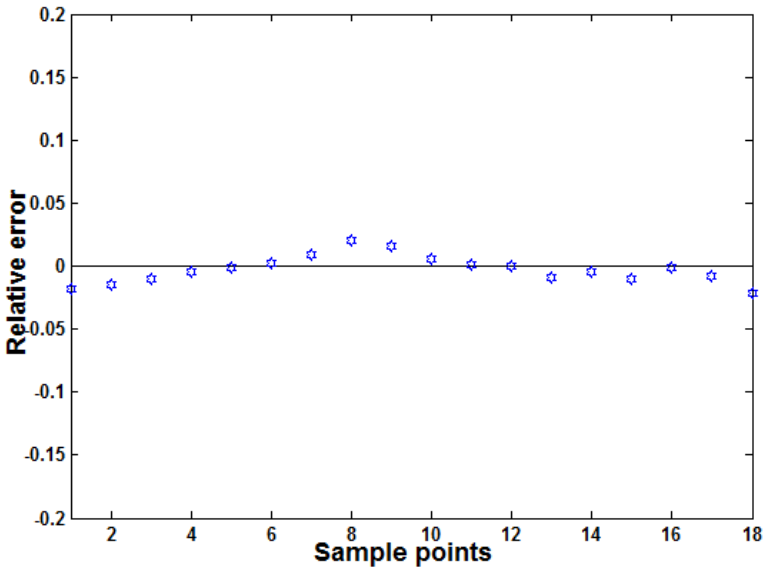

Fig.5 The relative error

\section{Conclusion}

In this paper, the method of grid search is applied to optimize SVM parameters, and quantitatively analyze the concentration range of $1 \% \sim 25 \%$ of the standard $\mathrm{CO}_{2}$ gas, with advantages of fast convergence and high model prediction accuracy. Absolute error of simulation models is less than $4 \%$, in line with national testing standards on emissions and completely meet the actual demand of gas measurement. Therefore, method of grid search combined with SVM has a certain potential for development and mining space in gas quantitative analysis modeling in the infrared spectrum of $\mathrm{CO}_{2}$ gas. 


\section{References}

[1] Cong-Ming Qiao.Quantitative Analysis of Tri-component Gas Mixture Based on PLS-SVR. Journal of Taiyuan University of Technology, 2014, 01:120-122+ 127.

[2] Xin Bao ,Lian-Kui Dai .Spectral Quantitative Analysis Based on Local Least Square Support Vector Machine Regression.Chinese Journal of Analytical Chemistry,2008,36(1):75.

[3] Jian-Guo Liu,Zhen-Tao An,Qian Zhang. Analysis of Mixed Inflammable Gases Based on Sensor Array and RBF Network.Equipment Environmental Engineer- ing, 2013,03: 113-116.

[4] Bao-Ping Tang,Wen-Yi Liu ,Yong-Hua Jiang. Parameter optimized Morlet wavelet de-noising method based on cross validation method.Journal of Chongqing University, 2010,01:1-6.

[5] Xing-Ling Wang,Zhan-Bing Li. Identifying the Parameters of the Kernel Function in Support Vector Machines Based on the Grid-Search Method.Journal of Ocean University of Qingdao, 2005,35 (5): 859-862. 